\title{
A LICENSE PLATE RECOGNITION SYSTEM IN E-GOVERNMENT
}

\author{
Hsien-Chu WU, Chwei-Shyong TSAI, and Ching-Hao LAI
}

\begin{abstract}
In recent years, there has been a lot of research in license plate recognition, and many license plate recognition systems have been proposed and used. In the past, the license plate recognition system was a key element in many applications such as traffic control and parking lots access control. In this article, the authors present current research in license plate recognition, and elaborate on the use of license plate recognition in e-Government. License plate recognition involves three main components: plate detection, character segmentation and character recognition. The authors consider all three components and thoroughly deal with the issue how to apply license plate recognition in e-Government in order to improve performance.
\end{abstract}

Keywords: e-Government, License Plate Recognition, Plate Detection, Character Segmentation, Traffic Control.

Nowadays, license plate recognition is extensively used in traffic management to identify a car whose owner has violated traffic laws or to find stolen vehicles. It is also applied to parking lot access control. Or, in other words, parking lots do not need human resources. When a car enters a parking lot, a computer equipped with a sensor and a license plate recognition system can recognize the license plate number of the car, and record the car data and the entry time. When the car leaves the parking place, the computer can automatically compute the parking cost. The license plate recognition system is automated and convenient. In addition, it is cost efficient due to the fact that less human resources are needed.

The license plate recognition system could also be used in e-Government. E-Government can apply license plate recognition to car identification. In recent years, a lot of cameras have been installed at the intersections by government or police. These cameras record a lot of images. If license plate recognition systems are installed at police offices, networks can be used to connect the cameras and the police offices. The cameras capture images regularly. When a camera captures an image, it can be 
transferred to the police office and the license plate number can be identified by the license plate recognition system. This license plate number can be used to acquire any information about the car and record the license plate number, time, place and so on. This information can aid the police in seizing car thieves and in traffic control. In addition, there have been numerous parking lots in the city areas. Usually, more than one person has to be employed at a given parking lot to record license plate number and to compute parking costs. If license plate recognition systems are installed at the parking lots, the license plate numbers of all cars parked in parking lots can be identified and the parking costs computed automatically. If license plate recognition systems are installed at government parking lots, the government can employ fewer employees and save more costs on employment.

License plate recognition systems provide to e-Government convenience and efficiency. In this article, recent research on license plate recognition is presented and some directions how to apply the methods of license plate recognition and to enable license plate recognition systems to work efficiently in an e-Government environment are given.

\section{Overview}

License plate recognition consists of three main phases: license plate detection, character segmentation and character recognition. The role of license plate detection is to extract the license plate regions from a vehicle image. Sometimes, there may be more than one license plates in an image. Some research in the field of license plate detection deals with the issue how to extract multiple license plates from an image. It is a very important subject in license plate recognition. After extracting all license plates from an image, all characters in a license plate have to be segmented at the character segmentation stage of license plate recognition. If the obtained character segment is good, character recognition will be more accurate. The final stage completed by any license plate recognition system is character recognition. At this stage, all characters have to be recognized. This stage is the most important one in license plate recognition. It has a substantial effect on success. In this section, the authors survey recent research on license plate recognition systems.

\section{License Plate Detection}

There has been a lot of research in license plate detection. There exist two principal types of image sources in plate detection. One is a video image and the other is a static image. If the image source is video, one has to extract the single license plate in real time. Due to the fact that it is necessary to extract the license plate in a very short time, it is extremely important that we extract the license plate quickly. If the source 
image is static, extracting multiple license plates from an image with a complex background is the main issue.

In the method proposed by Kim and Chien, Generalized Symmetry Transform (GST) and edge detection are used for license plate detection and normalization. ${ }^{1}$ Park and co-workers use a neural network for license plate detection. ${ }^{2}$ These methods are applied to images with only one car and without complex background.

There are many works that deal with images with a complex background. It is very difficult to extract many license plates from an image with a complex background. Gao and Zhou proposed a method to extract the license plates from an image with a complex background. ${ }^{3}$ They use histogram equalization to find a threshold to enhance the license plate image. After image enhancement, the authors compute the variance of the pixels in every large region. There would be some noise in these regions, and there may not be license plate regions. Gao and Zhou then smooth the edges by image dilation. Finally, they check the number of pixels, the proportion of width and height, and the proportion of black pixels in the region. Their method then eliminates the regions that are not license plate regions and outputs the images of license plates. Gao and Zhou assume that the license plates are near the center of the images. When the license plate is in the margins of the image, the license plate would not be extracted easily. The accuracy of Gao and Zhou's method is $80.7 \%$.

Maro, Chacon and Zimmernam use Pulse Coupled Neural Network (PCNN) to find the candidate regions from a static image that consist of only one car. ${ }^{4}$ Then, they use statistics and edge detection to find the regions of license plates and output them. There are three features in this technique. First, the images are static. Second, only one license plate from an image is extracted. Third, it is difficult to extract license plate from an image with a complex background.

\section{Character Segmentation}

In license plate recognition, when all license plate images are extracted from an image, one has to find all characters in the license plate and recognize them. Character segmentation is applied to the license plate in order to outline the individual characters. It affects greatly the accuracy of recognition. If the contours of the characters are inaccurate, it might lead to errors in the recognition stage or even to failed recognition stage.

Majority of current research deals with the segmentation of only one type of license plates. The methods proposed use prior knowledge to help in character segmentation. Prior knowledge includes information such as the size of the license plate, the size of a character, the size of the interval between the characters, and the number of all characters within a license plate. If there is only one type of license plates, it is useful 


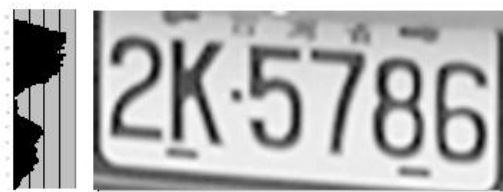

(a)

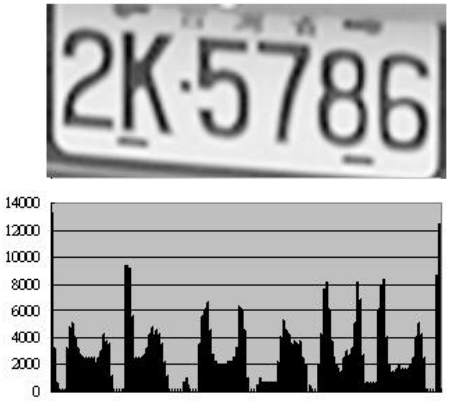

(b)

Figure 1: (a) Vertical Projection; (b) Horizontal Projection.

to make use of prior knowledge in character segmentation to achieve better results.

The character segmentation methods proposed in the majority of works are quite straightforward. They are typically based on the projection method..$^{5}$ The projection method uses vertical and horizontal projections to perform vertical and horizontal segmentation, respectively. Horizontal segmentation eliminates the unnecessary top and bottom parts of the license plate image. And vertical segmentation divides each character that is in the same license plate image. The projection method analyzes the vertical and horizontal projections (shown in Figure 1). How to locate all the valleys is a very important part of the whole process due to the fact that the valleys usually occur in the intervals between the characters within a license plate image. The process of locating valleys in the projection is called "projection analysis." Segmentation lines are created at these valleys. The projection method is the simplest, the most traditional, and most commonly used method for character segmentation. Rahman, Badawy and Radmanesh use only the projection method to segment all characters. ${ }^{6}$ However, if the projection method is used alone, the problems of rivet, rotation, and illumination variance can be observed. When these problems appear, there will be many errors in character segmentation. Zhang, Yang and Wang use also projection method to segment characters. ${ }^{7}$ In addition, they use prior knowledge and Hough transform to help in segmenting the characters. Prior knowledge is the information about license plate in China, and Hough transform can help in deleting wrong horizontal segmentation lines and combining the correct lines. Before the segmentation, the authors divide the license plate image into several parts and perform horizontal and vertical segmentations, respectively, for each part. Zhang-Yang-Wang's method can solve the problems of rivet, rotation, and illumination variance. Their method could be used in the license plate recognition systems in e-Government. 


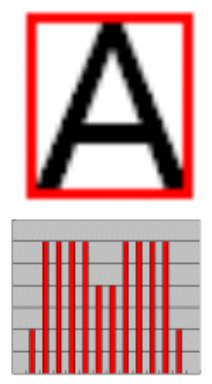

(a)

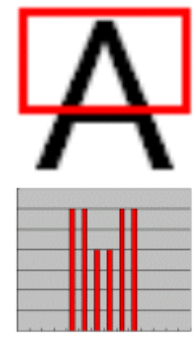

(b)

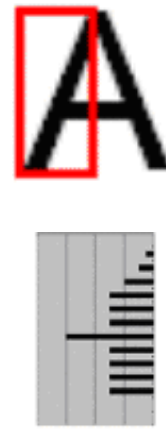

(c)

Figure 2: (a) Horizontal and Full-Size Histogram; (b) Upper-Half Histogram; (c) Left-Half Histogram.

\section{Character Recognition}

The final and the most important stage of license plate recognition is character recognition. At this stage, the character images that are extracted from the license plate image have to be recognized. The research on character recognition distinguishes between several main methods: methods using character features, methods based on neural networks, methods using pattern mapping, etc. Prior to formally applying a neural network, one has to generate many samples of characters to train the network. It is essential to know how to form the inputs to the neural network and how to train it. Pattern mapping is a traditional method. Before recognizing characters, the patterns have to be created. There are several types of patterns: images of each character, of different sizes or at different angles, projections of each character, and others.

The method proposed by Rahman and colleagues is based on pattern mapping. ${ }^{8}$ There are fifteen different types of projection histograms for each character in a pattern database before applying character recognition. These fifteen histograms are created using different parts of the characters ${ }^{9}$ : (1) full-size (horizontal); (2) lower-half (horizontal); (3) upper-half (horizontal); (4) lower-one-third (horizontal); (5) upper-onethird (horizontal); (6) lower-one-forth (horizontal); (7) upper-one-forth (horizontal); (8) upper-two-third (horizontal); (9) full-size (vertical); (10) left-half (vertical); (11) right-half (vertical); (12) left-one-third (vertical); (13) right-one-third (vertical); (14) left-one-fourth (vertical); (15) right-one-fourth (vertical). Figure 2 illustrates horizontal and full-size histogram (Figure 2(a)), upper-half histogram (Figure 2(b)) 
and left-half histogram (Figure 2(a)). Histogram mapping is performed until a single character is recognized.

Koval and co-workers use a neural network with the objective to take advantage of the network capability to solve the problems with noise and different positions of characters on a license plate. ${ }^{10}$ They use images of characters to train the neural network. The structure of a general neural network is shown in Figure 3. The neural network is multi-layered and has 663 elements. The experimental results demonstrate accuracy of $95 \%$ for plate images having noise with $50 \%$ density.

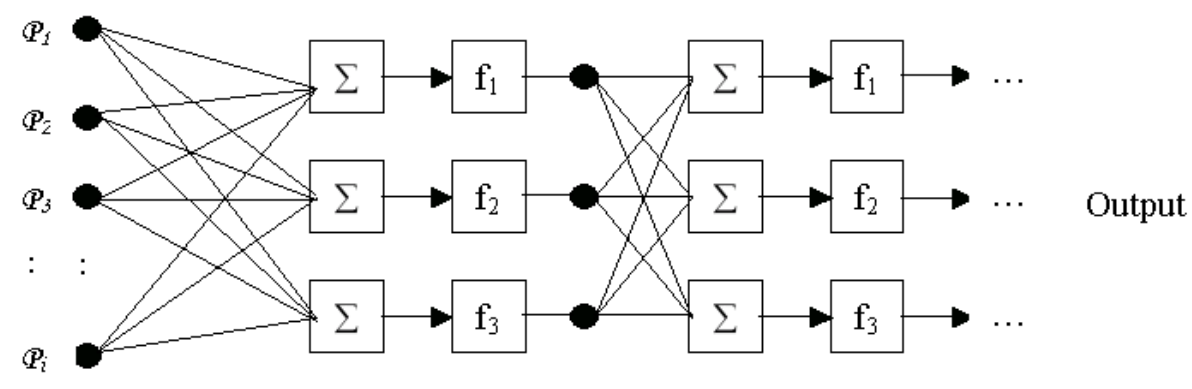

Input

Layer 1

Layer 2

Figure 3: General Structure of a Neural Network.

Dai, Ma, Liu and Li divide character recognition into three stages: preprocessing, feature extraction, and classification. ${ }^{11}$ There are three steps in preprocessing: noise removal, character normalization and changing all pixel values to binary values. These steps can help in improving the quality of character images and they are beneficial for feature extraction. There are three features to be extracted including projection feature (CPF), perimeter distance feature (PDF) and direction contribution density (DCD). The extracted features are used then to classify the characters. All techniques in the Dai-Ma-Liu-Li's method are very simple, and the performance is satisfactory. The accuracy is above $97 \%$ in daytime and $90 \%$ at night. The recognition time is between 0.1 and 1.5 seconds.

Wang and Lee extract two features before character recognition to help in recognizing the characters. ${ }^{12}$ These features are "crossing-count features (CCFs)" and "peripheral background area features (PBAFs)." In the preceding phase, the input character image has to be normalized in a coordinate system. Please note that the images of the same character may be of different type. Three different types of the character "A" are given in Figure 4. In principle, CCFs and PBAFs are very useful in character recognition. The accuracy of Wang and Lee's method is $98.6 \%$, and it is very accurate. 


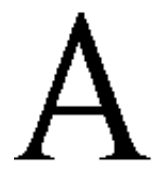

(a)

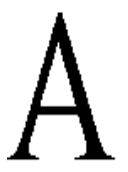

(b)

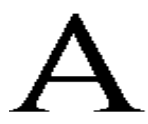

(c)

Figure 4: Different Types of Character "A".

\section{Discussion}

Many methods for license plate recognition have been proposed. However, different methods satisfy different requirements and have different applications. In this section, the authors will elaborate on the issue which methods are suitable for achieving high performance in e-Government.

As mentioned above, the license plate recognition system can be used in e-Government for traffic control, parking lots access control and so forth. However, the license plate recognition system has to work in real time. In order to develop an on-line, high speed license plate recognition system in e-Government, cameras have to be installed on roads or at the entrances of parking lots outdoors, as well as license plate recognition system has to be installed at control centers. Networks can be used to connect each camera and control center. When a camera captures a car image, the image can be transferred to the control center. The license plate recognition system installed at the control center can recognize the image and output the license plate number. It can record the license plate number, time, location, etc. These records are very useful. At the police offices, they can be used to obtain the traffic flow. The police can also access information about a stolen car and arrest the car thieves quickly. At parking lots, these records can be used to compute the costs for parking.

\section{License Plate Detection}

In this section, the authors propose a method for license plate detection in e-Government. In e-Government, license plate recognition has to be fast and very accurate. License plate detection is used for finding and extracting license plate regions in a car image. In general, the pixel values in the license plate region are very different due to the fact that the characters and the background of the license plate have a big difference in pixel values. We can find the region that has the biggest difference in pixel values, or the license plate region in the image. This method is very common. To aid 


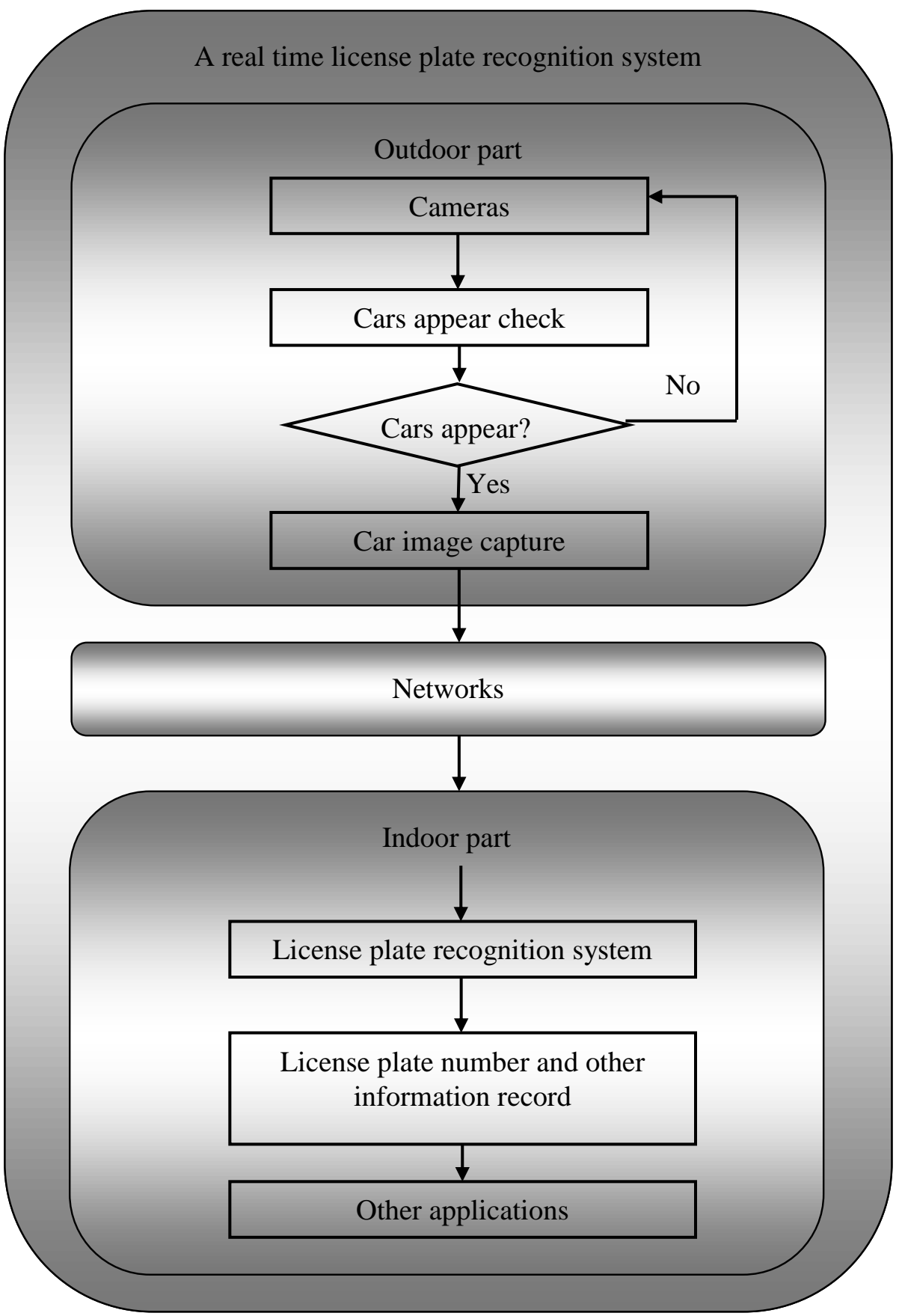

Figure 5: Structure of the Proposed License Plate Recognition System. 


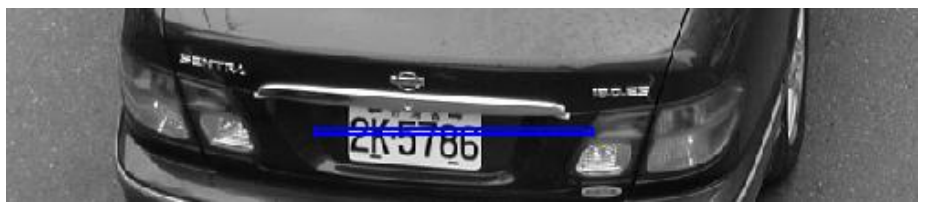

(a)

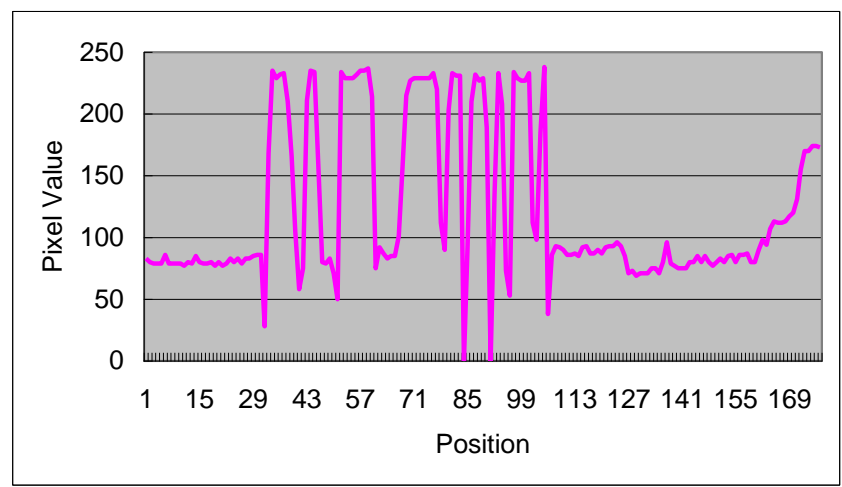

(b)

Figure 6: (a) The License Plate; (b) Horizontal Histogram.

license plate recognition in e-Government, the authors propose the following modification: the department issuing license plates could put a special logo on the license plate. When the license plate recognition system extracts license plates, the system can locate the special logo first. The method of finding license plates will be simpler and faster.

\section{Character Segmentation}

In general, the research on character segmentation often uses some prior information, such as the size of characters, the intervals between characters, and the size of the license plates. Using this prior information, the characters in the license plate can be segmented quickly and precisely.

Considering the fact that the characteristics of the license plates are useful in segmenting the characters, the standard for license plates have to be unified. If all license plates have a common standard, the license plate recognition system can segment characters derived from different types of license plates. 
As elaborated above, a simple and fast method for character segmentation is the projection method. Using horizontal and vertical projection histograms, one can locate segmentation points. These points may be incorrect, and one can use the characteristics of the license plates to eliminate such points. Horizontal and vertical projection histograms are shown in Figure 1. We recommend this method, because it is rather simple and fast.

\section{Character Recognition}

Pattern mapping is a common and simple method for character recognition. In a nutshell, many patterns for each character are created in the database. Once a character image is extracted and segmented, it is compared with all patterns in the database. There are several types of patterns, including images of each character, projection histograms ${ }^{13}$ of each character, etc. Using projection histograms to perform character recognition is quicker. The authors recommend using projection histograms to produce patterns in the database.

In applying pattern mapping methods, the size and the font of the characters are very important. In order to get better recognition results, the patterns have to be created using the same font of the characters in the license plates. The size of characters is also very important. Before proceeding with the mapping work, all character images have to be normalized, and the size has to be the same as that of the pattern.

Some letters look similar to numbers, for example: $\mathrm{B}$ and 8; I and 1; $\mathrm{O}$ and $0 ; \mathrm{S}$ and 5 ; etc. If letters and numbers have designated positions on the license plate- for example, there are 7 characters on a license plate, and the first three characters contain only letters and the remainder contains numbers-letters and numbers will not be mixed. Therefore, the authors recommend that letter and number positions be divided on a license plate.

\section{Conclusions}

License plate recognition systems can help government in traffic control, in seizing car thieves and in managing no-man parking lots automatically. Therefore, in order to develop an efficient e-Government, the license plate recognition system will be very useful.

In this article, the authors have presented the state-of-the-art research on license plate recognition. There has been a great number of methods proposed for license plate detection, character segmentation and character recognition. In order to be applied in e-Government, the license plate recognition system has to be a quicker and more accurate real-time system. It is recommended that the methods of license plate recognition be simple. 
There is no doubt that the license plate recognition system will be used by the government more and more in the future and, therefore, it is very important to design improved and more efficient systems for application in an e-Government environment.

\section{Notes:}

1 Dong-Su Kim and Sung-I. Chien, “Automatic Car License Plate Extraction Using Modified Generalized Symmetry Transform and Image Warping" (paper presented at the IEEE International Symposium on Industrial Electronics ISIE 2001, Pusan, Korea, 12-16 June 2001), Volume 3, 2022-2027.

2 Sung Han Park, Kwang In Kim, Keechul Jung, and Hyung Jin Kim, "Locating Car License Plates Using Neural Networks," IEE Electronics Letters 35, no. 17 (August 1999): 14751477.

3 Da-Shan Gao and Jie Zhou, "Car License Plates Detection from Complex Scene" (paper presented at the International Conference on Signal Processing, Beijing, 21-25 August 2000), 1409-1414.

4 I. Maro, M. Chacon, and S. Alejandro Zimmerman, "License Plate Location based on a Dynamic PCNN Scheme" (paper presented at the IEEE International Symposium on Computational Intelligence in Robotics and Automation, 16-20 July 2003), 972- 976.

5 Yungang Zhang and Changshui Zhang, "A New Algorithm for Character Segmentation of License Plate" (paper presented at the IEEE Intelligent Vehicles Symposium, Beijing, 9-11 June 2003), 106-109.

6 Choudhury A. Rahman, Wael M. Badawy, and Ahmad Radmanesh, “A Real Time Vehicle’s License Plate Recognition System" (paper presented at the IEEE Conference on Advanced Video and Signal Based Surveillance (AVSS'03), Miami, Florida, 21-22 July 2003), (IEEE Computer Society, 2003), 163-166.

7 Zhang, Yang, and Wang, “A New Algorithm for Character Segmentation of License Plate."

8 Rahman, Badawy, and Radmanesh, "A Real Time Vehicle's License Plate Recognition System."

9 Rahman, Badawy, and Radmanesh, "A Real Time Vehicle's License Plate Recognition System."

${ }^{10}$ Valeriy N. Koval, Volodymyr Turchenko, V. Kochan, Anatoly Sachenko, and George Markowsky, "Smart License Plate Recognition System based on Image Processing Using Neural Network" (paper presented at the Second IEEE International Workshop on Intelligent Data Acquisition and Advanced Computing System: Technology and Applications, 8-10 September 2003), 123-127. 
${ }^{11}$ Yan Dai, Hongqing Ma, Jilin Liu, and Langang Li, "A High Performance License Plate Recognition System based on the Web Technique" (paper presented at the IEEE Intelligent Transportation Systems Conference, Oakland, CA, USA, 25-29 August 2001), 325-329.

${ }^{12}$ Shen-Zheng Wang and Hsi-Jian Lee, "Detection and Recognition of License Plate Characters with Different Appearances" (paper presented at the Sixth IEEE Intelligent Transportation Systems, Shanghai, China, 12-15 October 2003), 979-984.

${ }^{13}$ Rahman, Badawy, and Radmanesh, "A Real Time Vehicle's License Plate Recognition System."

HSIEN-CHU WU was born in Tainan, Taiwan, Republic of China, on 26 October 1962. She received B.S. and M.S. degrees in Applied Mathematics in 1985 and 1987, respectively, from the National Chung Hsing University, Taichung, Taiwan. She received her Ph.D. in Computer Science and Information Engineering in 2002 from the National Chung Cheng University, Chiayi, Taiwan. From 1987 to 2002, she was a lecturer of the Department of Information Management at National Taichung Institute Technology, Taichung, Taiwan. Since August 2002, she has worked as an associate professor at the Department of Information Management of National Taichung Institute Technology, Taichung, Taiwan. Her research interests include image authentication, digital watermarking and image processing. Address for correspondence: Department of Information Management, National Taichung Institute of Technology, 129 Sec. 3, San-Min Road, Taichung, Taiwan 404, R.O.C. FAX: 886-422196610;Email:wuhc@ntit.edu.tw.

CHWEI-SHYONG TSAI was born in Changhua, Taiwan, Republic of China, on 3 September 1962. He received a B.S. degree in Applied Mathematics from National Chung Hsing University, Taichung, Taiwan, in 1984. He also received a M.S. degree in Computer Science and Electronic Engineer from National Center University, Chungli, Taiwan, in 1986. $\mathrm{He}$ received his Ph.D. degree in Computer Science and Information Engineering in 2002 from National Chung Cheng University, Chiayi, Taiwan. From August 2002 until 2004, he was an associate professor at the Department of Information Management at National Taichung Institute of Technology, Taichung, Taiwan. Since August 2004, he has been an associate professor at the Department of Management Information System at National Chung Hsing University, Taichung, Taiwan. His research interests include image authentication, information hiding, and cryptography. Address for correspondence: Department of Management Information System, National Chung Hsing University, 250, Kuo-Kuang Road, Taichung, Taiwan 402, R.O.C.Email: tsaics@ntit.edu.tw.

CHING-HAO LAI was born in Taichung, Taiwan, R.O.C., in 1982. He received a B.B.A. degree from the Department of Information Management of National Taichung Institute of Technology, Taichung, Taiwan, R.O.C., in 2004. He is currently working towards a M.S. degree at the Institute of Computer Science and Information Technology of National Taichung Institute of Technology, Taichung, Taiwan, R.O.C. His research interests include image processing and pattern recognition. E-mail: s18933111@ntit.edu.tw. 